\title{
General case of reinforced concrete rod elements calculation using the diagram method
}

\author{
Oleg Radaykin ${ }^{1}$, Linar Sabitov², Leysan Akhtyamova ${ }^{3}$, and Timur Arakcheev \\ ${ }^{1}$ Energoprogress Engineering center LLC, 420044, Kazan, Russian Federation \\ ${ }^{2}$ Kazan state power engineering University, 420066, Kazan, Russian Federation \\ ${ }^{3}$ Don state technical University, 3440002, Rostov-on-Don, Russian Federation
}

\begin{abstract}
Abstarct. In BC 63.13330 the general calculation case for the diagram method is oblique off-center compression, which takes into account only three components of internal force factors in the cross section: the longitudinal force $-\mathrm{N}_{z}-$ and two bending moments relative to the corresponding axes $-\mathrm{M}_{\mathrm{x}}$ and $\mathrm{M}_{\mathrm{y}}$. The other three components-the $\mathrm{Qx}$ and $\mathrm{Q}_{\mathrm{y}}$ transfer forces and the $\mathrm{M}_{\mathrm{z}}$ torque - are left out of consideration. In addition, for this case, the search in the available literature, including the founders of the diagram method, for the output of calculation formulas, was not successful - in all sources they are given in ready-made form without evidence. This article is intended to try filling in these gaps. For this purpose, based on the expressions for rod displacements that are generally accepted in mechanics, in particular on the Mora integral of displacements, the resolving expressions of the diagram method are obtained in the most general form.
\end{abstract}

\section{Introduction}

BC 63.13330 also includes the diagrammatic method as an alternative to the ultimate force method for the reinforced concrete rods' design. Its distinctive feature lies in the possibility of calculating for both groups of limiting states according to the same formulas, as well as obtaining a stress-strain state (SSS) of the considered elements at all loading stages: from zero to failure. In this set of rules, the general design case for this method is oblique offcenter compression, which takes into account only three components of internal force factors in the section: longitudinal force - Nz - and two bending moments about the corresponding axes $-M_{x}$ and $M_{y}$. The other three components are shearing forces $Q_{x}$ and $Q_{y}$ and torque $M_{z}$ - remain out of consideration. In addition, for oblique eccentric compression, the search in the available literature, including the founders of the diagram method [1] - [3], the calculation formulas' derivation was not crowned with success - in all sources they are already presented in a finished form without proof. This article is intended to try filling in these gaps.

\section{Models and Methods}

$$
\varepsilon_{z}=\varepsilon_{z}^{(1)}+\varepsilon_{z}^{(2)}+\varepsilon_{z}^{(2)}=\varepsilon_{0}+\chi_{y} x-\chi_{x} y
$$




$$
\begin{gathered}
\sigma_{z}=E \varepsilon_{z}=E\left(\varepsilon_{0}+\chi_{y} x-\chi_{x} y\right) \\
N_{z}=\int_{A} \sigma_{z} d A=E\left(A \varepsilon_{0}+\chi_{y} S_{y}-\chi_{x} S_{x}\right) \\
M_{x}=-\int_{A} \sigma_{z} y d A=E\left(-S_{x} \varepsilon_{0}-\chi_{y} I_{x y}+\chi_{x} I_{x}\right) \\
M_{y}=\int_{A} \sigma_{z} x d A=E\left(S_{y} \varepsilon_{0}+\chi_{y} I_{y}-\chi_{x} I_{x y}\right)
\end{gathered}
$$

In the special case of oblique eccentric compression, the governing equations for the rod shown in Fig. 1, a, are known. For example, in [4] such expressions are given:

$$
\left\{\begin{array}{l}
N_{z} \\
M_{x} \\
M_{y}
\end{array}\right\}=E\left[\begin{array}{ccc}
A & -S_{x} & S_{y} \\
-S_{x} & I_{x} & -I_{x y} \\
S_{y} & -I_{x y} & I_{y}
\end{array}\right] \times\left\{\begin{array}{l}
\varepsilon_{z} \\
\chi_{x} \\
\chi_{y}
\end{array}\right\}=[D] \times\left\{\begin{array}{l}
\varepsilon_{z} \\
\chi_{x} \\
\chi_{y}
\end{array}\right\}
$$

where $\varepsilon_{z}$ is a longitudinal relative deformations of the rod; $\chi_{x}, \chi_{y}$ are the curvature of the longitudinal axis of the rod relative to the corresponding axes; $E$ is the material deformation modulus; $A, S_{x}, S_{y}, I_{x}, I_{y}, I_{x y}$ show the geometric characteristics of the section: area, static moments, moments of inertia, centrifugal moment of inertia.

If the adopted coordinate axes coincide with the main central ones, then the static moments are equal to zero $S_{x}, S_{y}$ and centrifugal moment of inertia $I_{x y}$, and the stiffness matrix $[D]$ turns into a diagonal one.

For a reinforced concrete section, the formula (1) can be rewritten as:

$$
\left\{\begin{array}{l}
N_{z} \\
M_{y} \\
M_{x}
\end{array}\right\}=\left[\begin{array}{ccc}
D_{11} & -D_{12} & D_{13} \\
-D_{21} & D_{22} & -D_{23} \\
D_{31} & -D_{32} & D_{33}
\end{array}\right] \times\left\{\begin{array}{l}
\varepsilon_{z} \\
\chi_{x} \\
\chi_{y}
\end{array}\right\}=[D] \times\left\{\begin{array}{l}
\varepsilon_{z} \\
\chi_{x} \\
\chi_{y}
\end{array}\right\},
$$

where for the section shown in Fig. 1b, the components of the stiffness matrix are:

$$
\begin{aligned}
& D_{11}=\sum_{i=1}^{n-1} \sum_{j=1}^{m-1} v_{b, i j} E_{b, i j} A_{b, i j}+\sum_{k=1}^{p} v_{s, k} E_{s, k} A_{s, k}, \\
& D_{12}=D_{21}=\sum_{i=1}^{n-1} \sum_{j=1}^{m-1} v_{b, i j} E_{b, i j} A_{b, i j} y_{b i}+\sum_{k=1}^{p} v_{s, k} E_{s, k} A_{s, k} y_{s, k}, \\
& D_{13}=D_{31}=\sum_{i=1}^{n-1} \sum_{j=1}^{m-1} v_{b, i j} E_{b, i j} A_{b, i j} x_{b i}+\sum_{k=1}^{p} v_{s, k} E_{s, k} A_{s, k} x_{s, k}, \\
& D_{22}=\sum_{i=1}^{n-1} \sum_{j=1}^{m-1} v_{b, i j} E_{b, i j} A_{b, i j} y_{b i}^{2}+\sum_{k=1}^{p} v_{s, k} E_{s, k} A_{s, k} y_{s, k}^{2}, \\
& D_{23}=D_{32}=\sum_{i=1}^{n-1} \sum_{j=1}^{m-1} v_{b, i j} E_{b, i j} A_{b, i j} x_{b i} y_{b i}+\sum_{k=1}^{p} v_{s, k} E_{s, k} A_{s, k}^{m-1} v_{b, i j} E_{b, i j} A_{b, i j} x_{b i}^{2}+\sum_{k=1}^{p} v_{s, k} E_{s, k} A_{s, k} x_{s, k}^{2},
\end{aligned}
$$


where $v_{b}, v_{s}$ are the coefficients of the secant modulus of deformation (according to Karpenko N.I.) or the coefficients of elastic-plastic deformations (according to Murashev V.I.), respectively, of concrete and reinforcement; the rest of the (geometric) values are clear from Fig. 1, b.

Comparing the obtained expressions with the formulas (8.39) - (8.47) BC 63.13330, we find a lot of similarities. The difference between the set of rules is, firstly, in the rotation of the coordinate axes $O x y$ about the axis $O z$ on $90^{\circ}$ clockwise, and secondly, in the complete absence of minus signs in the stiffness matrix, which is apparently connected with the rotation of the axes. The third difference lies in the indexing of bending moments: in this article, the generally accepted in the resistance of materials is used - the subscript at the moment denotes the coordinate axis relative to which rotation occurs (for example, the moment $M_{x}$ causes rotation about the axis $O x$ ); in BC 63.13330 - otherwise: the subscript at the moment indicates the coordinate axis along which the moment acts. At the same time, although the plane in which a particular moment acts is concretized in $\mathrm{BC}$, without this specification, rotation relative to any of the two remaining axes can occur, which causes confusion. Therefore, in our works we use the designations generally accepted in the strength of materials and structural mechanics.
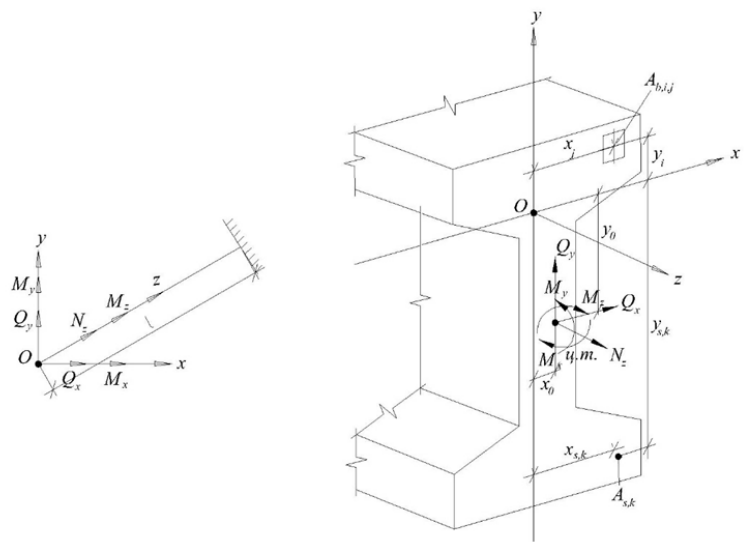

Fig. 1. Design scheme: $a$ - rod; $b$ - cross section

The solution to the system of equations (2) will be:

$$
\left\{\begin{array}{l}
\varepsilon_{z} \\
\chi_{x} \\
\chi_{y}
\end{array}\right\}=\left[\begin{array}{ccc}
D_{11} & -D_{12} & D_{13} \\
-D_{21} & D_{22} & -D_{23} \\
D_{31} & -D_{32} & D_{33}
\end{array}\right]^{-1} \times\left\{\begin{array}{l}
N_{z} \\
M_{y} \\
M_{x}
\end{array}\right\}=[D]^{-1} \times\left\{\begin{array}{c}
N_{z} \\
M_{y} \\
M_{x}
\end{array}\right\},
$$

To derive the general case of calculating the rod (Fig. 1, a) by the diagrammatic method, we write the Mohr displacement integral [4]:

$$
\Delta_{1 P}=\int_{0}^{l}\left(\frac{\bar{N}_{z 1} N_{z P}}{D_{z}}+\frac{\bar{M}_{x 1} M_{x P}}{D_{x}}+\frac{\bar{M}_{y 1} M_{y P}}{D_{y}}+\frac{\bar{M}_{z 1} M_{z P}}{D_{k}}+k_{x} \frac{\bar{Q}_{x 1} Q_{x P}}{D_{Q}}+k_{y} \frac{\bar{Q}_{y 1} Q_{y P}}{D_{Q}}\right) d z
$$

The formulas are valid if at the pole $O$ the rod is set to the right coordinate system Oxyz: axis $z$-along the rod, axis $x$ - from drawing plane, axis $y$ - up. Moreover, the axes are the main central ones. The positive direction for the moments and angles of rotation is 
counterclockwise as viewed from the positive end of that axis. For compactness, the moments and angles of rotation are designated as spin vectors.

Let us consider each of the six movements separately. So, in the action $\bar{N}_{z 1}=1$ and $N_{z}$ (the rest of the force factors are equal to zero), using the rule of multiplying the diagrams, we obtain a displacement along the axis $O z: u_{z}=\int_{0}^{l} \frac{N_{z}}{D_{z}} d z$. Pole rotation Orod relative to the axis $O x$ at the corner $\varphi_{x}$ possible both from the action of the moments $\bar{M}_{x 1}=1, M_{x}-$ they will cause displacement $\varphi_{x 1}=\int_{0}^{l} \frac{M_{x}}{D_{x}} d z$, and from the action of cutting forces $\bar{Q}_{y 1}=1, Q_{y}$, which create a moment equal $Q_{y} z$ and the corresponding movement $\varphi_{x 2}=-\int_{0}^{l} \frac{Q_{y} z}{D_{x}} d z$. Then $\varphi_{x}=\varphi_{x 1}+\varphi_{x 2}=\int_{0}^{l} \frac{M_{x}}{D_{x}} d z-\int_{0}^{l} \frac{Q_{y} z}{D_{x}} d z$. The rest of the displacement components are obtained by analogy:

$$
\begin{gathered}
u_{z}=\int_{0}^{l} \frac{N_{z}}{D_{z}} d z, \varphi_{x}=\int_{0}^{l} \frac{M_{x}}{D_{x}} d z-\int_{0}^{l} \frac{Q_{y} z}{D_{x}} d z, \varphi_{y}=\int_{0}^{l} \frac{M_{y}}{D_{y}} d z+\int_{0}^{l} \frac{Q_{x} z}{D_{y}} d z, \varphi_{z}=\int_{0}^{l} \frac{M_{z}}{D_{k}} d z, \\
u_{x}=\int_{0}^{l} k_{x} \frac{Q_{x}}{D_{Q}} d z+\int_{0}^{l} \frac{Q_{x} z^{2}}{D_{y}} d z+\int_{0}^{l} \frac{M_{y} z}{D_{y}} d z, u_{y}=\int_{0}^{l} k_{y} \frac{Q_{y}}{D_{Q}} d z+\int_{0}^{l} \frac{Q_{y} z^{2}}{D_{x}} d z-\int_{0}^{l} \frac{M_{x} z}{D_{x}} d z,
\end{gathered}
$$

where: $D_{z}$ is the axial stiffness of the rod, $D_{x}, D_{y}$ - flexural, $D_{Q}-$ shear, $D_{k}-$ twisting; $l$-rod length; $k_{x}, k_{y}$ are the shear shape factors (for a rectangular section $k_{x}=k_{y}=1,2$ ).More details about the stiffness will be given at the end of the article.

To find the shape coefficients during shear, the following expressions are used (reinforcement is neglected):

$$
k_{x}=\frac{A_{b}}{I_{b y}} \int_{A_{b}} \frac{S_{b y}^{o m c} d A_{b}}{b^{2}}, k_{y}=\frac{A_{b}}{I_{b x}} \int_{A_{b}} \frac{S_{b x}^{o m c} d A_{b}}{b^{2}}
$$

where $A_{b}$ shows the concrete section area; $b$ is a concrete section width; $I_{b x}, I_{b y}$ are the moments of inertia of a concrete section relative to the axes $O x$ and $O y ; S_{b x}^{o m c}, S_{b y}^{o m c}$ define the static moments of the cut-off part of the concrete section relative to the corresponding axes.

The vector of forces applied at the rod pole $O$, we write it in the form:

$$
\{P\}^{T}=\left\{\begin{array}{llllll}
N_{z} & Q_{x} & Q_{y} & M_{z} & M_{x} & M_{y}
\end{array}\right\}^{T},
$$

the corresponding displacement vector:

$$
\{u\}^{T}=\left\{\begin{array}{llllll}
u_{z} & u_{x} & u_{y} & \varphi_{z} & \varphi_{x} & \varphi_{y}
\end{array}\right\}^{T}
$$




\section{Research results and their analysis}

In the expressions (6), we differentiate along the coordinate $z$ linear displacements $u_{z}$ once: $\frac{\partial u_{z}}{\partial z}=\varepsilon_{z}$, linear displacements $u_{x}, u_{y}$ - twice: $\frac{\partial^{2} u_{y}}{\partial z^{2}}=\chi_{x}, \frac{\partial^{2} u_{x}}{\partial z^{2}}=\chi_{y}$, angles of rotation $\varphi_{z}$, $\varphi_{x}, \varphi_{y}$ - once: $\frac{\partial \varphi_{z}}{\partial z}=\theta_{z}, \frac{\partial \varphi_{x}}{\partial z}=\chi_{x}, \frac{\partial \varphi_{y}}{\partial z}=\chi_{y}$, where $\theta_{z}$ is a relative angle of twist about the axis $\mathrm{Oz}$. Now we get:

$$
\begin{gathered}
\varepsilon_{z}=\frac{N_{z}}{D_{z}}, \\
\chi_{x}=k_{y} \frac{q_{y}}{D_{Q}}+\frac{q_{y} z^{2}}{D_{x}}+\frac{2 Q_{y} z}{D_{x}}-\frac{Q_{y} z}{D_{x}}-\frac{M_{x}}{D_{x}}=q_{y}\left(\frac{k_{y}}{D_{Q}}+\frac{z^{2}}{D_{x}}\right)+\frac{Q_{y} z}{D_{x}}-\frac{M_{x}}{D_{x}}, \\
\chi_{y}=k_{x} \frac{q_{x}}{D_{Q}}+\frac{q_{x} z^{2}}{D_{y}}+\frac{2 Q_{x} z}{D_{y}}+\frac{Q_{x} z}{D_{y}}+\frac{M_{y}}{D_{y}}=q_{x}\left(\frac{k_{x}}{D_{Q}}+\frac{z^{2}}{D_{y}}\right)+\frac{3 Q_{x} z}{D_{y}}+\frac{M_{y}}{D_{y}}, \\
\theta_{z}=\frac{M_{z}}{D_{k}}, \chi_{x}=-\frac{Q_{y} z}{D_{x}}+\frac{M_{x}}{D_{x}}, \chi_{y}=\frac{Q_{x} z}{D_{y}}+\frac{M_{y}}{D_{y}} .
\end{gathered}
$$

In these formulas, we make the replacement: $Q_{y} z=-M_{x}, Q_{x} z=M_{y}$, then we will have:

$$
\begin{gathered}
\varepsilon_{z}=\frac{N_{z}}{D_{z}}, \chi_{x}=q_{y}\left(\frac{k_{y}}{D_{Q}}+\frac{z^{2}}{D_{x}}\right)-\frac{2 M_{x}}{D_{x}}, \chi_{y}=q_{x}\left(\frac{k_{x}}{D_{Q}}+\frac{z^{2}}{D_{y}}\right)+\frac{4 M_{y}}{D_{y}}, \\
\theta_{z}=\frac{M_{z}}{D_{k}}, \chi_{x}=-\frac{M_{y}}{D_{x}}+\frac{M_{x}}{D_{x}}, \chi_{y}=\frac{M_{x}}{D_{y}}+\frac{M_{y}}{D_{y}} .
\end{gathered}
$$

The equations 2, 3 and, accordingly, 5,6 in the formulas (11) are identical - they determine one and the same quantity, therefore we discard, for example, 2 and 3 . We obtain the following system of equations:

$$
\varepsilon_{z}=\frac{N_{z}}{D_{z}}, \theta_{z}=\frac{M_{z}}{D_{k}}, \chi_{x}=\frac{M_{x}-M_{y}}{D_{x}}, \chi_{y}=\frac{M_{y}+M_{x}}{D_{y}} .
$$

Or in a matrix form:

$$
\left\{\begin{array}{c}
\varepsilon_{z} \\
\theta_{z} \\
\chi_{x} \\
\chi_{y}
\end{array}\right\}=\left[\begin{array}{cccc}
\frac{1}{D_{z}} & 0 & 0 & 0 \\
0 & \frac{1}{D_{z}} & 0 & 0 \\
0 & 0 & \frac{1}{D_{x}} & -\frac{1}{D_{x}} \\
0 & 0 & \frac{1}{D_{y}} & \frac{1}{D_{y}}
\end{array}\right] \times\left\{\begin{array}{c}
N_{z} \\
M_{z} \\
M_{x} \\
M_{y}
\end{array}\right\},
$$


The expressions obtained do not contain shearing forces and the shifts are taken into account implicitly. To take them into account explicitly, it is necessary to discard the last two expressions in the formulas (11) and put $z=l, \quad q_{x}=\frac{3 Q_{x}}{8 l} \ldots \frac{5 Q_{x}}{8 l} \approx \frac{2 Q_{x}}{l}$, $q_{y}=\frac{3 Q_{y}}{8 l} \ldots \frac{5 Q_{y}}{8 l} \approx \frac{2 Q_{y}}{l}$. Then (11) will appear in the form:

$$
\begin{gathered}
\varepsilon_{z}=\frac{N_{z}}{D_{z}}, \theta_{z}=\frac{M_{z}}{D_{k}}, \chi_{x}=2 Q_{y}\left(\frac{k_{y}}{D_{Q} l}+\frac{l}{D_{x}}\right)-\frac{2 M_{x}}{D_{x}}, \\
\chi_{y}=2 Q_{x}\left(\frac{k_{x}}{D_{Q} l}+\frac{l}{D_{y}}\right)+\frac{4 M_{y}}{D_{y}} .
\end{gathered}
$$

Or in matrix form:

$$
\left\{\begin{array}{l}
\varepsilon_{z} \\
\theta_{z} \\
\chi_{x} \\
\chi_{y}
\end{array}\right\}=\left[\begin{array}{cccccc}
\frac{1}{D_{z}} & 0 & 0 & 0 & 0 & 0 \\
0 & 0 & 0 & \frac{1}{D_{z}} & 0 & 0 \\
0 & 0 & \frac{2 k_{y}}{D_{Q} l}+\frac{2 l}{D_{x}} & 0 & -\frac{2}{D_{x}} & 0 \\
0 & 0 & 0 & \frac{2 k_{x}}{D_{Q} l}+\frac{2 l}{D_{y}} & 0 & \frac{4}{D_{y}}
\end{array}\right] \times\left\{\begin{array}{l}
N_{z} \\
Q_{x} \\
Q_{y} \\
M_{z} \\
M_{x} \\
M_{y}
\end{array}\right\} .
$$

It should be noted that, although shear forces are taken into account in the expressions (14) and (15), in the deformation vector, shear deformations yare absent, they are taken into account indirectly through shear stiffness $D_{Q}$, which depends on $\tau$ and $\gamma$.

We also note that in the structure of the formulas for curvatures, the first term is responsible for the pure shear strains effect on the curvature, the second term is for bending deformations caused by a shearing force, and the third term is for bending deformations caused by the moment action.

In addition, the proposed approach carries the development prospects due to taking into account, in addition to longitudinal deformations $\varepsilon_{z}$ also transverse deformations $\varepsilon_{x}$ and $\varepsilon_{y}$. Possible options for such clarifications are given in the works [5], [6], [7].

Prior to this, all formulas were derived with respect to the main central axes of the rod. With an arbitrary choice of the coordinate system $O^{\prime} x^{\prime} y^{\prime} z^{\prime}$ it is necessary to make adjustments to the stiffness characteristics of the section based on the following reasoning (Fig. 1, b). The longitudinal force has no eccentricity relative to the main central axes, $a_{x}=a_{y}=0$, and the centrifugal moment of inertia and the corresponding centrifugal stiffness of the rod section are equal to zero: $I_{x y}=I_{b x y}+I_{s x y}=0, D_{x y}=0$. The last value is determined by the last expression from the formulas (3): $D_{x y}=D_{33}$.

The static moments about the axes are also equal to zero $O x$ and $O y$ and the corresponding static stiffnesses: $S_{x}=S_{b x}+S_{s x}=0$ and $S_{y}=S_{b y}+S_{s y}=0, \quad D_{S x}=0$ and $D_{S y}=0$. The specified stiffnesses are determined by the expressions 2 and 3 of the formulas (3): $D_{S x}=D_{12}=D_{21}, D_{S y}=D_{13}=D_{31}$. 
When specifying the coordinate system arbitrarily, it is necessary to take into account additional deformations of the section rotation caused by the eccentrically applied longitudinal force $N_{z}$, as well as additional turns from $M_{x}$ about the axis Oyand from $M_{y}$ about the axis $O x$. This is achieved by including the formulas (12) or (14) to the first expression, the relative deformations caused by bending: $\varepsilon_{z}=\varepsilon_{0}+\chi_{y} x-\chi_{x} y$, where $\varepsilon_{0}=\frac{N_{z}}{D_{z}}$. And, in addition, it is necessary to replace the bending stiffnesses with the following values:

$$
\begin{gathered}
D_{x}=\frac{D_{x}^{\prime}+D_{y}^{\prime}}{2}+\frac{1}{2} \sqrt{\left(D_{x}^{\prime}-D_{y}^{\prime}\right)^{2}+4 D_{x y}^{\prime}}-\frac{D_{S x}^{\prime 2}}{D_{z}^{\prime}}, \\
D_{y}=\frac{D_{x}^{\prime}+D_{y}^{\prime}}{2}-\frac{1}{2} \sqrt{\left(D_{x}^{\prime}-D_{y}^{\prime}\right)^{2}+4 D_{x y}^{\prime}}-\frac{D_{S y}^{\prime 2}}{D_{z}^{\prime}},
\end{gathered}
$$

where is the stroke ' $\left.^{\prime}\right\rangle-$ means that this value is calculated with respect to arbitrary axes $O^{\prime} x^{\prime} y^{\prime} z^{\prime}$.

Wherein $D_{z}=D_{z}^{\prime}, D_{Q}=D_{Q}^{\prime}$ и $D_{k}=D_{k}^{\prime}$.

It is also necessary to replace bending moments and shear forces with:

$$
\begin{gathered}
M_{x}=\frac{\left(M_{x}^{\prime}+N_{z} a_{y}\right) \cos \left(\alpha_{0 M}-\alpha\right)}{\cos \alpha_{0 M}}=\frac{N_{z}\left(e_{y}^{\prime}+a_{y}\right) \cos \left(\alpha_{0 M}-\alpha\right)}{\cos \alpha_{0 M}}=\frac{N_{z} e_{y} \cos \left(\alpha_{0 M}-\alpha\right),}{\cos \alpha_{0 M}}, \\
M_{y}=\frac{\left(M_{y}^{\prime}+N_{z} a_{x}\right) \sin \left(\alpha_{0 M}-\alpha\right)}{\sin \alpha_{0 M}}=\frac{N_{z}\left(e_{x}^{\prime}+a_{x}\right) \sin \left(\alpha_{0 M}-\alpha\right)}{\sin \alpha_{0 M}}=\frac{N_{z} e_{x} \sin \left(\alpha_{0 M}-\alpha\right)}{\sin \alpha_{0 M}}, \\
Q_{x}=\frac{Q_{x}^{\prime} \cos \left(\alpha_{0 Q}-\alpha\right)}{\cos \alpha_{0 Q}}, Q_{y}=\frac{Q_{y}^{\prime} \sin \left(\alpha_{0 Q}-\alpha\right)}{\sin \alpha_{0 Q}},
\end{gathered}
$$

where $a_{x}, a_{y}$ show distance between corresponding axes $O^{\prime} x^{\prime} y$ 'and $O x y$ (taken with their own sign relative to the axes $O x y) ; e_{y}^{\prime}=\frac{M_{x}^{\prime}}{N_{z}}, e_{x}^{\prime}=\frac{M_{y}^{\prime}}{N_{z}}-$ axis projection $O^{\prime} x^{\prime} y^{\prime}$ eccentricity of longitudinal force application relative to the center $O^{\prime} ; e_{y}=\frac{M_{x}}{N_{z}}, e_{x}=\frac{M_{y}}{N_{z}}$ is an axis projection $O x y$ eccentricity of application of longitudinal force relative to the center $O ; \alpha=\frac{1}{2} \operatorname{arctg}\left(-\frac{2 D_{x y}^{\prime}}{D_{x}^{\prime}-D_{y}^{\prime}}\right)$ is a rotation angle of the axes $O^{\prime} x^{\prime} y^{\prime}$ with respect to the axes $O x y$; $\alpha_{0 M}=\operatorname{arctg}\left(\frac{M_{y}^{\prime}}{M_{x}^{\prime}}\right) ; \quad \alpha_{0 Q}=\operatorname{arctg}\left(\frac{Q_{y}^{\prime}}{Q_{x}^{\prime}}\right)$ the angles between the vectors $M^{\prime}$ and $Q^{\prime}$ and horizontal axis $O^{\prime} x^{\prime}$ respectively.

For brevity, we denote $C_{M}=\frac{\cos \left(\alpha_{0 M}-\alpha\right)}{\cos \alpha_{0 M}}, \quad S_{M}=\frac{\sin \left(\alpha_{0 M}-\alpha\right)}{\sin \alpha_{0 M}}, \quad C_{Q}=\frac{\cos \left(\alpha_{0 Q}-\alpha\right)}{\cos \alpha_{0 Q}}$, $S_{Q}=\frac{\sin \left(\alpha_{0 Q}-\alpha\right)}{\sin \alpha_{0 Q}}$ 
Thus, for the formulas (12) we will have:

$$
\begin{gathered}
\varepsilon_{z}=\frac{N_{z}}{D_{z}}+\chi_{y} x-\chi_{x} y, \theta_{z}=\frac{M_{z}}{D_{k}}, \\
\chi_{x}=\frac{N_{z}\left(-e_{x}^{\prime}-a_{x}+e_{y}^{\prime}+a_{y}\right) C_{M}}{\frac{D_{x}^{\prime}+D_{y}^{\prime}}{2} \pm \frac{1}{2} \sqrt{\left(D_{x}^{\prime}-D_{y}^{\prime}\right)^{2}+4 D_{x y}^{\prime}}-\frac{D_{S x}^{\prime 2}}{D_{z}}}, \\
\chi_{y}=\frac{N_{z}\left(e_{x}^{\prime}+a_{x}+e_{y}^{\prime}+a_{y}\right) S_{M}}{\frac{D_{x}^{\prime}+D_{y}^{\prime}}{2} \mp \frac{1}{2} \sqrt{\left(D_{x}^{\prime}-D_{y}^{\prime}\right)^{2}+4 D_{x y}^{\prime}}-\frac{D_{S y}^{\prime 2}}{D_{z}}} .
\end{gathered}
$$

And we rewrite the formulas (14) as follows:

$$
\begin{gathered}
\varepsilon_{z}=\frac{N_{z}}{D_{z}}+\chi_{y} x-\chi_{x} y, \theta_{z}=\frac{M_{z}}{D_{k}}, \\
\chi_{x}=\frac{2 Q_{y}^{\prime} C_{Q} k_{y}}{D_{Q} l}+\frac{2 Q_{y}^{\prime} C_{Q} l-2 N_{z}\left(e_{y}^{\prime}+a_{y}\right) C_{M}}{\frac{D_{x}^{\prime}+D_{y}^{\prime}}{2} \pm \frac{1}{2} \sqrt{\left(D_{x}^{\prime}-D_{y}^{\prime}\right)^{2}+4 D_{x y}^{\prime}}-\frac{D_{S x}^{\prime 2}}{D_{z}}}, \\
\chi_{y}=\frac{2 Q_{x}^{\prime} S_{Q} k_{x}}{D_{Q} l}+\frac{2 Q_{x}^{\prime} S_{Q} l+4 N_{z}\left(e_{x}^{\prime}+a_{x}\right) S_{M}}{\frac{D_{x}^{\prime}+D_{y}^{\prime}}{2} \mp \frac{1}{2} \sqrt{\left(D_{x}^{\prime}-D_{y}^{\prime}\right)^{2}+4 D_{x y}^{\prime}}-\frac{D_{S y}^{\prime 2}}{D_{z}}} .
\end{gathered}
$$

Let us consider a special case when the angle $\alpha=0$, that is, the axes $O^{\prime} x^{\prime} y^{\prime}$ parallel $O x y$. It means that $\cos \alpha=1, D_{x y}^{\prime}=0$, then for (18) we will have:

$$
\begin{gathered}
\varepsilon_{z}=\frac{N_{z}}{D_{z}}+\chi_{y} x-\chi_{x} y, \theta_{z}=\frac{M_{z}}{D_{k}}, \chi_{x}=\frac{N_{z}\left(-e_{x}^{\prime}-a_{x}+e_{y}^{\prime}+a_{y}\right)}{D_{x}^{\prime}-\frac{D_{S x}^{\prime 2}}{D_{z}}}, \\
\chi_{y}=\frac{N_{z}\left(e_{x}^{\prime}+a_{x}+e_{y}^{\prime}+a_{y}\right)}{D_{y}^{\prime}-\frac{D_{S y}^{\prime 2}}{D_{z}}} .
\end{gathered}
$$

And for (19):

$$
\begin{gathered}
\varepsilon_{z}=\frac{N_{z}}{D_{z}}+\chi_{y} x-\chi_{x} y, \theta_{z}=\frac{M_{z}}{D_{k}}, \chi_{x}=\frac{2 Q_{y} k_{y}}{D_{Q} l}+\frac{2 Q_{y} l-2 N_{z}\left(e_{y}^{\prime}+a_{y}\right)}{D_{x}^{\prime}-\frac{D_{S x}^{\prime 2}}{D_{z}}}, \\
\chi_{y}=\frac{2 Q_{x} k_{x}}{D_{Q} l}+\frac{2 Q_{x} l+4 N_{z}\left(e_{x}^{\prime}+a_{x}\right)}{D_{y}^{\prime}-\frac{D_{S y}^{\prime 2}}{D_{z}}} .
\end{gathered}
$$


If the axes $O^{\prime} x^{\prime} y^{\prime}$ now move to the center of the section gravity, then $D_{S x}^{\prime}=0$, $D_{S y}^{\prime}=0, a_{x}=0, a_{y}=0, e_{x}^{\prime}=e_{x}, e_{y}^{\prime}=e_{y}, D_{x}^{\prime}=D_{x}, D_{y}^{\prime}=D_{y}, \varepsilon_{z}=\varepsilon_{0}$ then we get the formulas (12) and (14).

It should be noted that the expressions (12), (14), (18) - (21) were obtained for the first time.

Let us supplement them with the boundary conditions in the form of deformation criteria for the onset of a particular limiting state. So, the cracks formation in the element occurs when the maximum deformation in the most stretched concrete fiber reaches:

$$
\varepsilon_{b t}^{\max }=\varepsilon_{b t 2}
$$

When an element is destroyed, three cases are possible:

$$
\begin{gathered}
\varepsilon_{b}^{\max }=\varepsilon_{b 2}-\text { fragile destruction, } \\
\varepsilon_{s}^{\max }=\varepsilon_{s 0}-\text { "Plastic" destruction, } \\
\varepsilon_{b}^{\max }=\varepsilon_{b 2} \mathrm{U} \varepsilon_{s}^{\max }=\varepsilon_{s 0}-\text { destruction of the optimal section. }
\end{gathered}
$$

Let us list the advantages of the proposed approach:

1 - all six components of external force factors acting on the rod element are considered (in BC 63.13330 there are only three: $\left.N_{z}, M_{x}, M_{y}\right)$ : the main vector of forces $R-$ three $\left(N_{z}\right.$, $\left.Q_{x}, Q_{y}\right)$, at the main point $M-$ three $\left(M_{x}, M_{y}, M_{z}\right)$;

2 - the calculations take into account the work of the stretched zone of concrete above the crack;

3 - to describe the relationship between stresses and deformations of concrete, in addition to the diagrams of deformation for uniaxial tension / compression, shear diagrams are used $\langle\tau-\gamma\rangle$ [8], [9].Due to this, it becomes possible to calculate the cross-sections of bending elements in the joint action zone $M$ and $Q$ based on the diagrammatic method.

We will extend the proposed approach to a wider class of structural rod elements made of reinforced concrete. For this, we write down the formulas for determining stiffness in the most general form:

$$
\begin{aligned}
& D_{z}=\sum \sum v_{b} E_{b} A_{b}+\sum \sum k_{b, a d} v_{b, a d} E_{b, a d} A_{b, a d}+\sum k_{s} v_{s} E_{s} A_{s}+ \\
& +\sum k_{s, a d} v_{s, a d} E_{s, a d} A_{s, a d}+\sum \sum k_{r 1} v_{s, r 1} E_{s, r 1} A_{s, r 1}+\sum \sum k_{r 2} v_{s, r 2} E_{s, r 2} A_{s, r 2} \\
& D_{x}=\sum \sum v_{b} E_{b} I_{b x}+\sum \sum k_{b, a d} v_{b, a d} E_{b, a d} I_{b x, a d}+\sum k_{s} v_{s} E_{s} I_{s x}+ \\
& +\sum k_{s, a d} v_{s, a d} E_{s, a d} I_{s x, a d}+\sum \sum k_{r 1} v_{s, r 1} E_{s, r 1} I_{s x, r 1}+\sum \sum k_{r 2} v_{s, r 2} E_{s, r 2} I_{s x, r 2} \\
& D_{y}=\sum \sum v_{b} E_{b} I_{b y}+\sum \sum k_{b, a d} v_{b, a d} E_{b, a d} I_{b y, a d}+\sum k_{s} v_{s} E_{s} I_{s y}+ \\
& +\sum k_{s, a d} v_{s, a d} E_{s, a d} I_{s y, a d}+\sum \sum k_{r 1} v_{s, r 1} E_{s, r 1} I_{s y, r 1}+\sum \sum k_{r 2} v_{s, r 2} E_{s, r 2} I_{s y, r 2} \\
& D_{k}=\sum \sum v_{b} G_{b} I_{b k}+\sum \sum k_{b, a d} v_{b, a d} G_{b, a d} I_{b k, a d}+\sum k_{s} v_{s} G_{s} I_{s k}+ \\
& +\sum k_{s, a d} v_{s, a d} G_{s, a d} I_{s k, a d}+\sum \sum k_{r 1} v_{s, r 1} G_{s, r 1} I_{s k, r 1}+\sum \sum k_{r 2} v_{s, r 2} G_{s, r 2} I_{s k, r 2}
\end{aligned}
$$




$$
\begin{aligned}
& D_{Q}=\sum \sum v_{b} G_{b} A_{b}+\sum \sum k_{b, a d} v_{b, a d} G_{b, a d} A_{b, a d}+\sum k_{s} v_{s} G_{s} A_{s}+ \\
& +\sum k_{s, a d} v_{s, a d} G_{s, a d} A_{s, a d}+\sum \sum k_{r 1} v_{s, r 1} G_{s, r 1} A_{s, r 1}+\sum \sum k_{r 2} v_{s, r 2} G_{s, r 2} A_{s, r 2}
\end{aligned},
$$

where $E_{b}, E_{b, a d}, E_{s}, E_{s, a d}, E_{s, r 1}, E_{s, r 2}$ and $G_{b}, G_{b, a d}, G_{s}, G_{s, a d}, G_{s, r 1}, G_{s, r 2}$ are the initial deformation moduli and shear moduli, respectively, of the base concrete, added concrete reinforcement, main flexible rod reinforcement, added flexible rod reinforcement, rigid central reinforcement (steel core), rigid outer steel reinforcement (pipe shell); $A_{b}, A_{b, a d}$, $A_{s}, A_{s, a d}, A_{s, r 1}, A_{s, r 2}$ are the areas of the section corresponding components; $I_{b x}$, $I_{b x, a d}, I_{s x}, I_{s x, a d}, I_{s x, r 1}, I_{s x, r 2}$ and $I_{b x}, I_{b x, a d}, I_{s y}, I_{s y, a d}, I_{s y, r 1}, I_{s y, r 2}$ are the moments of inertia of the corresponding components of the section about the axes $O x$ and $O y$; $I_{b k}, I_{b k, a d}, I_{s k}, I_{s k, a d}, I_{s k, r 1}, I_{s k, r 2}$ are the torsional moments of inertia of the section corresponding components; $v_{b}, v_{b, a d}, v_{s}, v_{s, a d}, v_{s, r 1}, v_{s, r 2}$ are the secant modulus coefficients (otherwise, the elasto plastic deformation coefficients) of the corresponding components of the section; $k_{b, a d}, k_{s} k_{s, a d} k_{r 1} k_{r 2}$ are the coefficients of collaboration. Stiffness summation is performed over elementary areas (horizontally and vertically).

Further refinement of the proposed approach will consist in taking into account the actual mechanism of the appearance and development of the inclined [10] and spiral cracks [11], respectively, under the action of shear forces and torque, in determining the parameters of the rod section components' joint operation (partly studies on the current reinforcement of reinforced concrete beams with a steel-fiber-concrete "jacket" are made in [12] - [14]), taking into account the cyclic static and dynamic loads, which is important when designing wind turbines of the tower type [15]-[17].

\section{Conclusion}

1. The diagrammatic method continuity for calculating rod reinforced concrete elements under oblique eccentric compression according to BC 63.13330 and the classical approach of material strength have been established.

2. For the first time, the resolving equations of the diagrammatic method, in which all six components of internal force factors are taken into account, have been obtained. This is done based on Mohr's integral of displacements.

3. The proposed approach has development prospects. Its further refinement will consist in taking into account the actual mechanism of the appearance and development of inclined and spiral cracks, respectively, under the action of shear forces and torque, in determining the parameters of joint operation of the components of the cross section of the rod, taking into account cyclic static and dynamic loads, which is important in the tower type wind power plants' design.

\section{References}

1. V.N. Baykov, M.I. Dodonov, B.S. Rastorguev [et al.], Concrete and reinforced concrete 5, 16-18 (1987). 
2. N.I. Karpenko, T.A. Mukhamediev, M.A. Sapozhnikov, Improvement of methods for calculating statically indeterminate reinforced concrete structures, 1987.

3. N.I. Karpenko, General models of reinforced concrete mechanics (Stroyizdat, Moscow, 1996).

4. A.G. Gorshkov, V.N. Troshin, V.I. Shalashilin, Resistance of materials (Fizmatlit, Moscow, 2005).

5. N.I. Karpenko, Concrete and reinforced concrete 3, 14-16 (1989).

6. A.C. Silantev, Vestnik MGSU 2-1, 163-170 (2011).

7. E.D. Lazovsky, D.O. Glukhov, Bulletin of Polotsk state University. Ser. F, Applied Sciences 16, 33-39 (2013) (rus).

8. B.S. Sokolov, O.V. Radaykin, International journal for the calculation of civil and building structures 15 (3), 149-160 (2019).

9. N.I. Karpenko, B.S. Sokolov, O.V. Radaykin, Design of concrete, reinforced concrete, stone and reinforced stone elements and structures using diagram calculation methods: monograph (Publishing house of ASV, Moscow, 2019).

10. A.S. Zalesov, Yu.A. Klimov, Strength of reinforced concrete structures under the action of transverse forces (Budivelnyk, Kiev, 1989).

11. V.I. Kolchunov, A.I. Demyanov, M.I. Matveev, Construction and reconstruction 3 (89), 3-13 (2020).

12. O.V. Radaikin, L.A. Sharafutdinov, Izvestiya KGASU 1 (39), 111-120 (2017).

13. O.V. Radaikin, L.A. Sharafutdinov, Izvestiya KGASU 1 (47), 155-165 (2019).

14. O.V. Radaikin, L.A. Sharafutdinov, Bulletin of BSTU named after V. G. Shukhov 3, 34-45 (2020).

15. L. Mailyan, S. Yaziev, L. Sabitov, Y. Konoplev, O. Radaykin, Stress-strain state of the "combined tower-reinforced concrete foundation-foundation soil" system for high-rise structures, E3S Web of Conferences. Topical Problems of Green Architecture, Civil and Environmental Engineering, TPACEE 2019 (2020).

16. Yu.M. Strelkov, L.S. Sabitov, I.L. Kuznetsov, O.V. Radaykin, L.Sh. Akhtyamova, Computer modeling of the system" steel tower - reinforced concrete Foundation ground base " of wind power installations, Effective structures, materials and technologies in construction. Materials of the international scientific and practical conference (2019).

17. L.S. Sabitov, Yu.G. Konoplev, O.V. Radaykin, Bulletin of the Volgograd state University of architecture and civil engineering. Series: construction and architecture 1 (78), 345-355 (2020). 